International Journal of Clinical Pharmacology \& Toxicology (IJCPT)

ISSN 2167-910X

\title{
Liquid-Liquid Extraction vs Solid Phase Extraction in Biological Fluids and Drugs
}

Hideharu Shintani ${ }^{1^{*}}$

Chuo University, School of Science, Kasuga Bunkyo, Tokyo, Japan.

\section{*Corresponding Author:}

Hideharu Shintani

Chuo University, School of Science,

Kasuga Bunkyo, Tokyo, Japan.

Tel: +81425922336

E-mail: shintani@mail.hinocatv.ne.jp

Received: August 12, 2013

Published: August 30, 2013

Citation: Hideharu Shintani ( (2013) Liquid-Liquid Extraction vs Solid Phase Extraction in Biological Fluids and Drugs. Int J Clin Pharmacol Toxicol. 2(1e), 1. doi: http://dx.doi.org/10.19070/2167-910X-130004e

Copyright: Hideharu Shintani ${ }^{\odot}$ 2013. This is an open-access article distributed under the terms of the Creative Commons Attribution License, which permits unrestricted use, distribution and reproduction in any medium, provided the original author and source are credited.

Biological fluids have complicated matrix and it is quite hard task to analysis the interest free from admixtures in the matrix.

Traditionally liquid/liquid extraction (LLE) is employed for the analysis of biological compounds, based upon the manipulation of aqueous $\mathrm{pH}$ to extract drugs into organic solvent(s) with several sort of polar. This method is used on a variety of specimens including blood, urine, bile, gastric contents, and tissue homogenates and acidic, neutral, and alkaline drugs may be extracted in one analytical scheme. Knowing the chemical properties of the $\operatorname{drug}(\mathrm{s})$ of interest allows the proper selection of organic solvents to perform a successful extraction from a biological specimen and a further purification of the extracts can be attained with a back extraction. The disadvantage of LLE is that relatively large volumes of organic solvents are required, an evaporation step is required to concentrate the extracts prior to analysis, necessity of thermal stability of some thermal-fragile sample analysis for evaporation step, and LLE corresponds to only one theoretical plate solid phase extraction (SPE). Evaporation can be done using $\mathrm{N}_{2}$ gas blowing, but this procedure cannot utilize large volume of solvents.

SPE utilizes short length HPLC cartridge and deproteinazation is unnecessary. Blood sample can directly apply to SPE when using biological sample. The most superior point of SPE to LLE is that the theoretical number is more than 10,000 , indicating more than 10,000 times of LLE procedure s can be done in single SPE. In addition, volume of solvents used for SPE is quite small, almost all type of interest (hydrophobic to hydrophilic) can be applicable using hydrophilic column to hydrophobic column. The editor published many papers in Japanese and English up to 2000 using SPE and found out that SPE was superior to LLE. In addition, I want to strongly mention that the manual type SPE is quite inferior to automated SPE. This is because poor reproducibility of pressure to column for elution and so on. Therefore, reproducibility of recovery rates is quite low in case of manual SPE compared with automated SPE. As an automated SPE Benchmate $^{\mathrm{R}}$ and so on can be commercially available at $\$ 150,000$ with the combination of HPLC, PC, automated SPE and automated injector. This editorial notice is based on the editor's experience of the use of automated SPE. 